\title{
Complications Associated with Worse Visual Outcomes in Patients with Exudative Neovascular Age-Related Macular Degeneration
}

\author{
Costanza Barresi ${ }^{a, b}$ Enrico Borrellia, ${ }^{a}$ Federico Fantaguzzi ${ }^{a, b}$ \\ Domenico Grosso $^{a, b}$ Riccardo Sacconi ${ }^{a, b}$ Francesco Bandello ${ }^{a, b}$ \\ Giuseppe Querques ${ }^{\mathrm{a}, \mathrm{b}}$ \\ aVita-Salute San Raffaele University Milan, Milan, Italy; ${ }^{b}$ IRCCS San Raffaele Scientific Institute, Milan, Italy
}

\section{Keywords}

Age-related macular degeneration - Neovascular age-

related macular degeneration $\cdot$ Complications

\begin{abstract}
Background: Neovascular age-related macular degeneration (AMD) represents a leading cause of vision loss in older subjects. The introduction of anti-vascular endothelial growth factor therapy has enormously improved the visual prognosis in these patients. Summary: However, severe complications may still occur in this disease and their development may significantly affect short- and long-term visual outcomes. Key Messages: In this article, we provided a review of these complications, highlighting their distinctive signs or symptoms and focusing on the current evidence of treatment. To place the complications related to neovascular AMD in a proper context, this review deals with core components essential for proper comprehension of neovascular AMD that includes pathogenesis, clinical signs, and basic principles of treatment.

(c) 2021 S. Karger AG, Basel
\end{abstract}

\section{Introduction}

Age-related macular degeneration (AMD) is the leading cause of irreversible central vision loss among older people in developed countries [1]. Its prevalence is nearly $8.7 \%$, although this is expected to increase in the next few years due to exponential population aging. The projected number of people affected by AMD worldwide is $288 \mathrm{mil}-$ lion by 2040 [2].

AMD is often asymptomatic in the early stages which are characterized by the presence of drusen and/or retinal pigment epithelium (RPE) abnormalities. Conversely, severe central vision loss usually characterizes the late form of AMD: in the majority of cases (85\%), it is the result of macular neovascularization (MNV) development, while the remaining $15 \%$ is characterized by geographic atrophy $[3,4]$.

Noteworthy, progresses in AMD's diagnosis and therapy have brought a substantial paradigm shift in the management of neovascular exudative AMD. Many clinical trials proved that intravitreal inhibition of vascular endothelial growth factor (VEGF) may efficiently lessen pathophysiological processes, restore retinal morphology, and eventually preserve neurosensory function [5-10].

Correspondence to:

Giuseppe Querques, giuseppe.querques@ hotmail.it 
However, despite the impressive benefit of antiangiogenic therapy, it is still important to be aware that complications can occur, which may dramatically impact the treatment outcomes. Therefore, this review summarized main complications related to neovascular exudative AMD. Our aim was to provide a full description of its main complications, highlighting their distinctive signs or symptoms and focusing on the current evidence of treatment. To place the complications related to neovascular AMD in a proper context, this review begins with core components essential for proper comprehension of neovascular AMD that includes pathogenesis, clinical signs, and basic principles of treatment.

\section{Pathogenesis and Classification}

The presence of drusen and RPE pigmentary abnormalities represents the major hallmark of AMD. The exact pathophysiology of the disease is not fully understood. It is supposed to be the result of a multifactorial interaction between metabolic, functional, genetic, and environmental factors. It is widely recognized that the damage of the unit comprised of photoreceptor, RPE, Bruch's membrane, and choriocapillaris (CC) which play a key role in neovascular AMD pathogenesis $[11,12]$. Histopathological studies displayed that the RPE is primarily damaged in geographic atrophy, while MNV usually occurs in areas of CC impairment with viable RPE [13]. These areas of vascular dropout may result in RPE hypoxia, consequent increase of VEGF secretion, and stimulation of MNV growth.

Importantly, the term "MNV" (instead of "choroidal neovascularization") recognizes the fact that neovascularization does not necessarily originate from the choroid. MNV is indeed an invasion by vascular and associated tissues into the outer retina, subretinal space, or subRPE space in varying combinations [3].

The anatomic location of the neovascularization that may be determined by structural optical coherence tomography (OCT) imaging is used to subclassify the vascular component of the disease process. Type 1 and type $2 \mathrm{MNV}$ are characterized by an ingrowth of vessels from the CC into the sub-RPE and subretinal spaces, respectively. Conversely, type $3 \mathrm{MNV}$ refers to a neovascularization that originates from the retinal circulation, typically the deep capillary plexus, and grows toward the outer retina [3].

The presence of an exudative neovascular lesion has been historically considered the crucial element to define neovascular AMD [4]. However, assuming that type 1 MNV can be present without features of exudation, the term "exudative neovascular AMD" was actually suggested to be more accurate in order to exclude cases without exudation [3]. In detail, exudative neovascular AMD may be characterized by the pathological proliferation and exudation of type 1 , type 2 , or type $3 \mathrm{MNV}$.

\section{Clinical Aspects}

Patients with exudative neovascular AMD frequently complain of blurred vision and metamorphopsia, specially distorted near vision $[14,15]$. However, many patients may not complain of any symptoms or frequently describe only indefinite visual impairment. In these patients, decreased ability to perform functional tasks (e.g., face recognition) is strictly dependent on the visual acuity in the better-seeing eye [16]. Of note, the Amsler grid test may be useful for early detection of neovascular AMD [17]. Symptoms are generally secondary to intraretinal, subretinal, or sub-RPE fluid accumulation, hemorrhages, or damage of photoreceptors, and RPE in the setting of macular atrophy (MA) complicating neovascular AMD.

A careful ophthalmoscopic examination detects typical clinical signs of MNV: a pigment ring or a gray-green membrane, irregular elevation of the RPE, or a pigment epithelium detachment (PED), exudation, and/or blood. When the fibrotic component is prevalent, MNV may appear as a white or yellowish lesion. In the presence of RPE atrophy in the context of a type $1 \mathrm{MNV}$, the neovascular capillary network may become more visualizable. Nowadays, multimodal retinal imaging plays a key role in detecting early additional signs of MNV, all carefully reported by Spaide et al. [3] .

\section{Treatment}

To date, antiangiogenic therapy is considered as the gold standard of treatment for exudative neovascular AMD. Nowadays, several anti-VEGF drugs are available in clinical practice:

- Pegaptanib (Macugen; Eyetech/Pfizer) is an RNA oligonucleotide ligand that binds human $\mathrm{VEGF}_{165}$. It has historically represented the first FDA-approved intravitreal anti-VEGF therapy, but it has been supplanted by other more effective agents.

- Ranibizumab (Lucentis; Genentech) is an IgG1 kappa isotype monoclonal antibody fragment that binds 
VEGF. It was approved by FDA in 2007 and was the first treatment for exudative neovascular AMD offering a realistic expect to visual improvement.

- Bevacizumab (Avastin; Genentech) is also a full-length humanized monoclonal antibody targeting VEGF. Of note, Bevacizumab was approved in 2004 for the treatment of patients with colorectal cancer, and it has widely been used off-label for the intravitreal treatment of nAMD.

- Aflibercept (Eylea; Regeneron Pharmaceuticals) is a recombinant protein composed of the binding domains of 2 human VEGF receptors fused with the Fc region of IgG1.

- Brolucizumab (Beovu; Novartis Pharmaceutical Corporation) is a monoclonal antibody which targets the main VEGF A isoforms: $\mathrm{VEGF}_{110}, \mathrm{VEGF}_{121}$, and $\mathrm{VEGF}_{165}$.

The 3 widely used intravitreal anti-VEGF agents, ranibizumab, bevacizumab, and aflibercept, have proven to be highly effective treatments for preventing complications of neovascular AMD. However, mainly due to a compelling need for long-lasting therapy solutions, considerable research efforts continue toward developing extended-release therapeutics and intraocular drug-delivery devices that may become available soon.

Current treatment strategies include fixed-interval dosing or monthly visits with pro re nata (PRN - "as needed") re-treatment on evidence of exudative disease activity [18-20]. Noteworthy, even though the PRN regimen reduces the number of intravitreal injections by $3-4$ procedures in the first year of treatment, patients still sustain the same travel and visit burden as compared with the fixed regimen because of monthly follow-up visits [18-20].

Another approach named "treat and extend" (TREX, progressive extension of treatment and visit intervals up to 12 weeks depending on the clinical findings) is widely used. This regimen is aimed at minimizing total treatment burden by reducing both number of treatments and visits. The TREX regimen has been initially validated in several retrospective studies $[21,22]$ and noncomparative prospective trials $[23,24]$ that demonstrated that this regimen is noninferior to PRN or fixed dosing in terms of visual acuity gain. The Treat-and-Extend Protocol in Patients with Wet Age-Related Macular Degeneration (TREX-AMD) study was the first prospective, randomized trial which performed a comparison between monthly and TREX regimens in neovascular exudative AMD patients $[25,26]$. This trial enrolled 60 patients who were randomized to the monthly $(n=20)$ and TREX $(n=40)$

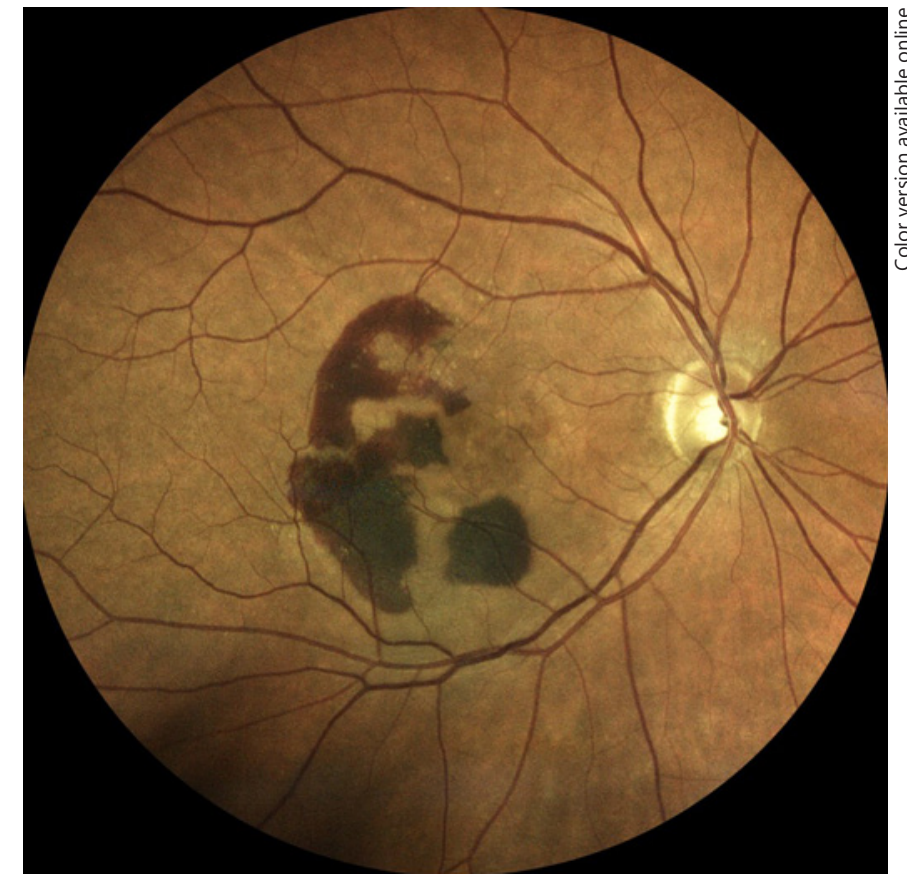

Fig. 1. Color fundus photograph from a patient with subretinal hemorrhage. The right eye of a patient with neovascular AMD complicated with massive subretinal hemorrhage. Color image shows SHM within the temporal and inferior macula. The patient was treated with anti-VEGF injections as the hemorrhage was outside the fovea. AMD, age-related macular degeneration; VEGF, vascular endothelial growth factor; SHM, massive subretinal hemorrhage.

cohorts. Mean visual acuity gains were comparable between the 2 regimens. In details, at the 1-year follow-up visit, the mean number of treatments was lower in the TREX cohort (13.0 vs. 10.1 injections, $p<0.0001$ ), while the gain in visual acuity was similar between the 2 groups [25]. Of note, after 2 years of follow-up, the gain in visual acuity did not significantly differ between the 2 groups (10.5 letters for the fixed-interval and 8.7 letters for the TREX groups, respectively) [26].

\section{Complications of Neovascular AMD}

\section{Massive Subretinal Hemorrhage}

Massive subretinal hemorrhage (SHM) is an uncommon but severe complication that may occur in the setting of exudative neovascular AMD (shown in Fig. 1). In a previous study, $17 \%$ of patients with neovascular AMD were reported to develop SMH during the course of their disease. However, large SMHs are likely to complicate 
only a small number of these patients - although the exact percentage has not been reported [27]. Patients complicated by SHM usually complain of progressive or sudden visual loss, according to the extent and thickness of the hemorrhage. Subretinal hemorrhages are often classified by their size, with massive SMHs defined as thick hemorrhages larger than 4 disc diameter extending beyond the temporal arcades [27].

While anticoagulant therapy represents a recognized risk factor for subretinal hemorrhage, the antiplatelet therapy as a risk factor for SHM is controversial. In a case series of patients with SHM, 19\% of subjects were in treatment with sodium warfarin or aspirin [28]. More importantly, in the CATT trial, an association between subretinal hemorrhage and anticoagulant or antiplatelet therapy was demonstrated to be significant only in patients with systemic hypertension [29]. However, the Macular Photocoagulation Study (MPS) reports did not display any association between the increased risk of hemorrhage and use of aspirin [30-32]. Assuming this, patients with AMD who are simultaneously treated with aspirin should not be at higher risk of SHM.

In the absence of treatment, the natural course of SHM is usually severe and progressive with a potential irreversible vision loss. A case series analyzing 60 eyes with large SHM (i.e., size superior to a disc area) showed that $80 \%$ of cases had a worsening in vision over a period of 24 months, with a mean final visual acuity of 20/1,250 Snellen [33]. Prognostic factors for final visual acuity include the SHM diameter and the time between SHM development and ophthalmology referral, as subretinal blood is known to rapidly damage the retina and RPE $[34,35]$.

Despite the wide range of techniques available for SHM management, there is still no consensus on the optimal approach [36]. For small-sized hemorrhages, the risk-benefit profile supports a more conservative approach with either anti-VEGF therapy alone [37] or in combination with pneumatic displacement with intravitreal tissue plasminogen activator (t-PA) $[38,39]$. Chen et al. [39] evaluated a total of 85,77 , and 81 eyes, respectively, at 1 week, 3 months, and 12 months after intravitreal tPA and expansible gas injection. Postoperatively, an improvement superior to 2 Snellen lines was achieved in $51 \%$ of the eyes at 1 week, $63 \%$ at 3 months, and $64 \%$ at 12 months. The main complications observed included breakthrough vitreous hemorrhage $(\mathrm{VH})(8 \%)$ and retinal detachment (3\%). For massive hemorrhages, current evidences support a treatment with vitrectomy in combination with subretinal t-PA and anti-VEGF, followed by gas tamponade and upright positioning $[40,41]$. In de-

Complications in Exudative Neovascular

Age-Related Macular Degeneration tails, Treumer et al. [40] evaluated short-term (3 months) and long-term (average of 17 months) visual outcomes with the latter treatment in a retrospective case series of 41 eyes. Visual acuity significantly improved with the latter treatment from preoperative values of $1.7-0.8$ to an average visual acuity of $0.9 \log M A R$. The encouraging results of t-PA, gas, and anti-VEGF therapy, with or without vitrectomy, justify further study and an attempt to standardize reporting. In particular, the following factors are usually considered for the treatment decision: (i) duration and area of SHM, (ii) foveal involvement, (iii) coagulation status, and (iv) the baseline BCVA [27]. Massive and foveal SHMs require more frequently prompt intervention.

\section{Vitreous Hemorrhage}

Dense breakthrough VH is a rare, but serious complication of submacular hemorrhage secondary to neovascular AMD with an estimated rate of 2-7\% [42]. Development of hemorrhage into the vitreous cavity may also be related to retinochoroidal shunts leading to bleeding from internal retinal vessels, photodynamic therapy treatment of subretinal new vessels, or blood from subretinal choroidal bleeding entering the vitreous cavity through microscopic retinal breaks [43].

Although the VH itself does not have a negative impact on visual function, abrupt and profound deterioration in visual acuity may occur because of the impediment to light to reach the retina. A prompt treatment with vitrectomy may be useful to eventually restore vision and accurately assess the retinal status. Although vitrectomy is known to be associated with a short-term improvement in visual acuity, the presence of a severe submacular hemorrhage may negatively impact on this improvement [44]. Furthermore, if extensive re-bleeding or scar formation occurs in the early postoperative period, patients should be advised that the treatment outcomes can be more unfavorable than expected [45]. The main risk factor for early scar formation is the extension of neovascular lesion and subretinal hemorrhage. Therefore, intravitreal injections of anti-VEGF agents may be helpful in preventing further hemorrhage and scarring in the cases of breakthrough $\mathrm{VH}$ secondary to submacular hemorrhage [46]. A recent study by Kim et al. [42] sought to evaluate the possible prognostic factors for patients with exudative AMD undergoing vitrectomy for breakthrough $\mathrm{VH}$. Univariate analysis revealed that older age (odds ratio [OR] 0.879; $p=0.007)$ and presence of massive submacular hemorrhage $(\mathrm{OR}, 0.081 ; p=0.022)$ were associated with a 

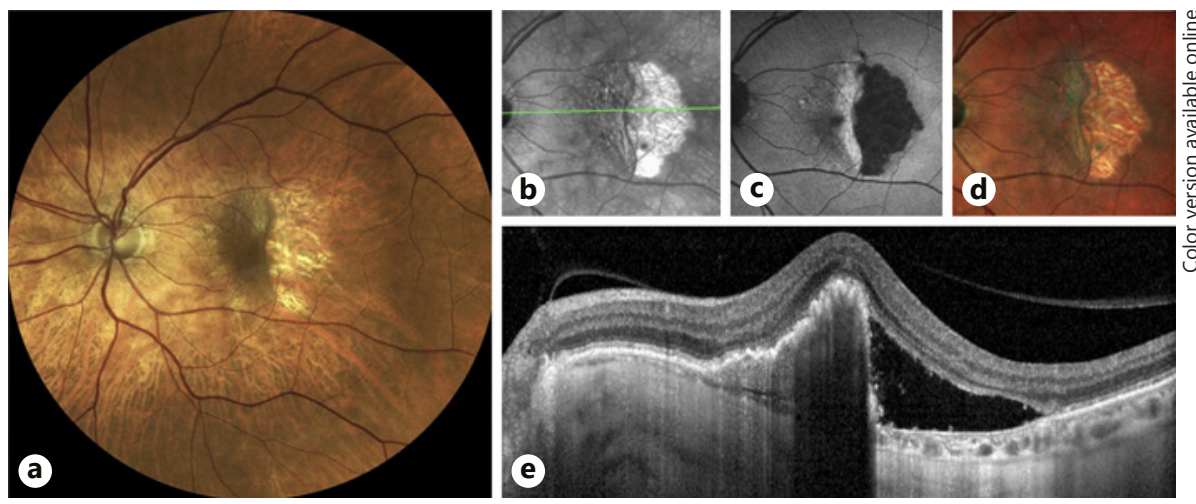

Fig. 2. Multimodal imaging from a patient with a retinal pigment epithelium tear. The left eye of a patient with neovascular AMD complicated with an RPE tear. a Color image shows a region of depigmented retina with exhibition of the underlying choroidal vessels and associated hyperpigmented areas that correspond to the rolled RPE. The green arrow in the near-IR reflectance image (b) illustrates the location and direction of the structural OCT-B scan (e) that demonstrates the presence of the RPE tear. c FAF of the RPE tear shows a hypoautofluorescent area in the region where the RPE denudes the choroid. $\mathbf{d}$ Multicolor image shows a region of depigmented retina with exhibition of the underlying choroidal

poorer 2-year visual outcome, while eyes with polypoidal choroidal vasculopathy (PCV) had a relatively more favorable visual outcome than nAMD eyes (OR, 0.137; $p=0.014)$. In details, the latter study identified 45 eyes undergoing vitrectomy due to $\mathrm{VH}$. Furthermore, these eyes were divided into 2 groups according to the presence of neovascular AMD versus PCV. At the final visit (39.9 months after surgery), the visual acuity was significantly better in the PCV group ( $p=0.003$ vs. neovascular AMD group) [45].

\section{Retinal Pigment Epithelium Tears}

RPE tears constitute a disruption of the RPE secondary to underlying tangential tensile forces (shown in Fig. 2, 3). While RPE tears may occur in the setting of a serous PED with or without evidence of contiguous MNV, these lesions may occasionally develop in eyes with exudative neovascular AMD without serous PED in the presence of large areas of hemorrhage [47-50]. Overall, the incidence of RPE tears ranges from 0.4 to $27 \%$ in cases of neovascular AMD [51, 52]. Notably, RPE tears usually occur in treatment-naive eyes or, eventually, they may complicate eyes soon after starting treatment, assuming that most RPE tears manifest within 3 months after the initiation of anti-VEGF therapy [53]. Risk factors for development of RPE tears include a PED height superior to 550 microns vessels and associated hyperpigmented areas that correspond to the rolled RPE. e SD-OCT shows an area of RPE disruption with integrity of the overlying retina. The RPE is interrupted at the temporal margin of the PED leaving the underlying Bruch's membrane and choroid exposed. A clear backscattering effect on the underlying choroid is evident where the RPE is absent. In the area where the RPE is present, there is a masking effect on the underlying choroid. AMD, age-related macular degeneration; RPE, retinal pigment epithelium; OCT, optical coherence tomography; FAF, fundus autofluorescence; PED, pigment epithelium detachment; IR, infrared.
[54], the presence of RPE microtears [52] or a subretinal cleft [55], and history of photodynamic therapy [48].

On funduscopy, RPE tears appear as regions of depigmented retina with exhibition of the underlying choroidal vessels and associated hyperpigmented lines that correspond to the rolled RPE. In RPE tears, fundus autofluorescence shows a hypoautofluorescent area in the region where the RPE denudes the choroid (shown in Fig. 2, 3). On fluorescein angiography, RPE tears have a peculiar aspect as the denuded choroid is characterized by an early hyperfluorescence, while the folded pigment epithelium is an obstacle for the fluorescence and this region is thus hypofluorescent. Importantly, evidence of leakage in the context of RPE tears suggests the presence of an underlying MNV. On structural OCT, RPE tears appear as areas of RPE disruption with integrity of the overlying retina. RPE tears are usually located at the PED's edges where a hyper-reflective signal corresponding to the broken and rolled RPE is usually displayed.

Noteworthy, RPE tears must be distinguished from RPE apertures. The latter develops in serous PEDs as single or multiple round discontinuities, not only at the base but also at the apex of a PED [56]. In contrast to RPE tears, RPE apertures are not characterized by rippling or retraction of the RPE [56]. 

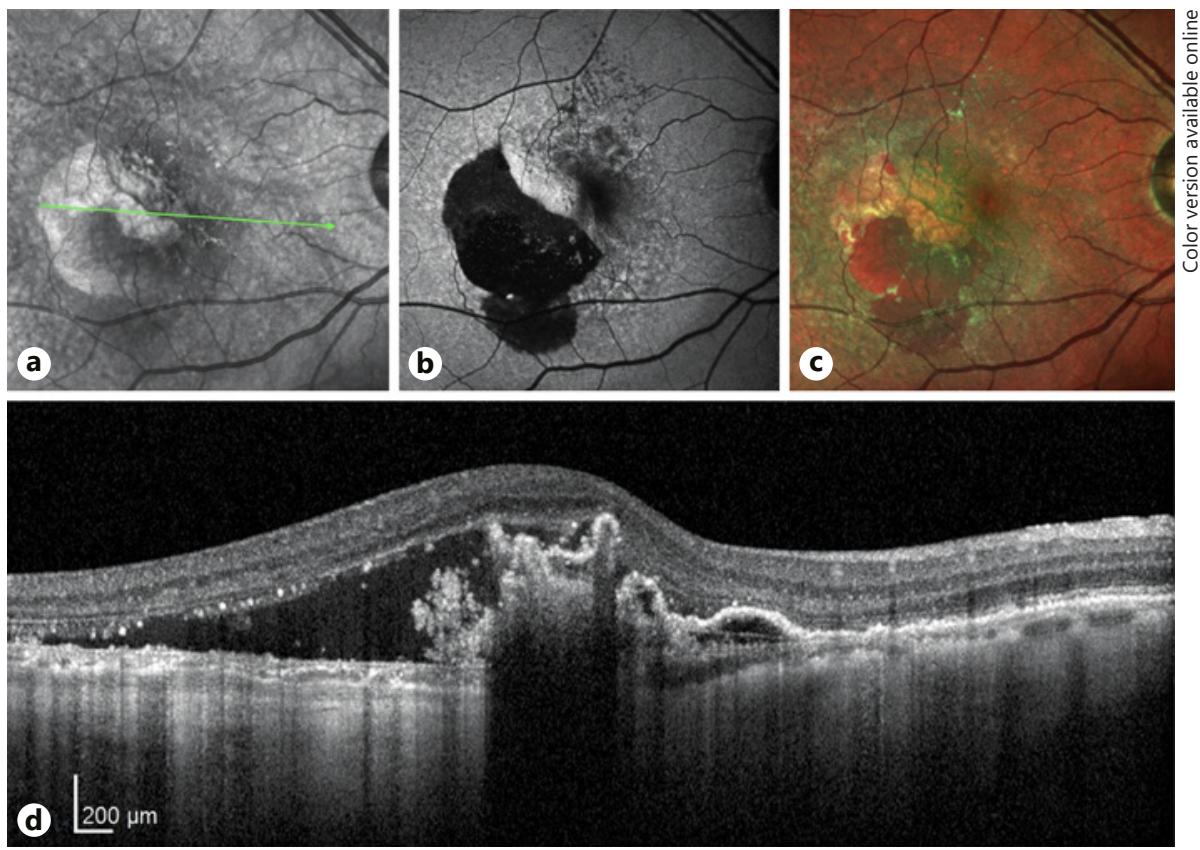

Fig. 3. Multimodal imaging from a patient with a retinal pigment epithelium tear. The right eye of a patient with neovascular AMD complicated with a RPE tear. The green arrow in the near-IR reflectance image (a) illustrates the location and direction of the structural OCT-B scan that demonstrates the presence of the RPE tear. b FAF of the RPE tear shows a hypoautofluorescent area in the region where the RPE denudes the choroid. c Multicolor image shows regions of depigmented retina with exhibition of the under- lying choroidal vessels and associated hyperpigmented lines that correspond to the rolled RPE. d SD-OCT shows an area of RPE disruption with integrity of the overlying retina. The tear is located at PED's edge where a hyper-reflective signal corresponding to the broken and rolled RPE is displayed. AMD, age-related macular degeneration; RPE, retinal pigment epithelium; OCT, optical coherence tomography; FAF, fundus autofluorescence; PED, pigment epithelium detachment; IR, infrared.
To date, there are no clinical practice guidelines on the management of RPE tears [51]. Anti-VEGF therapy has been proposed as the best therapeutic option to maintain the visual acuity of these patients. Sarraf et al. [57] reported on the use of anti-VEGF in patients with RPE tears. In their study cohort of 21 eyes, RPE tears were graded into 4 stages according to the morphological characteristics (i.e., greatest length in the vector direction of the tear and involvement of the fovea). The authors demonstrated that lower grade tears were more likely characterized by a better VA and better response to continued anti-VEGF therapy, as well as they were at lower risk of fibrosis development. Moreira et al. [58] also evaluated the mean VA in patients with RPE tears not involving the fovea reporting an improvement from 20/160 to 20/60 with additional anti-VEGF therapy over 52 months of follow-up.

\section{Macular Atrophy}

MA has been proposed as a general term to describe areas of photoreceptor and RPE loss in the macula whether it is associated with MNV or not (with the term "geo- graphic atrophy" being restricted for atrophy in absence of MNV) (shown in Fig. 4) [59, 60]. Assuming that MA represents a threatening complication of exudative neovascular AMD, a huge effort had been spent to better understand the pathophysiology of its development and its relationship with anti-VEGF treatment. At present, many potential nonexclusive mechanisms have been suggested to be involved in the occurrence of atrophy in eyes with exudative neovascular AMD.

First, atrophy was hypothesized to occur as a consequence of the AMD natural history and pathogenesis. Post hoc analyses of cohorts from large-scale clinical trials (HARBOR [61], CATT [62], and IVAN [20]) displayed that among eyes without detectable atrophy at baseline, 29.4, 18.3, and $29.7 \%$ of study eyes developed MA at the end of 2 years in the 3 studies, respectively. In the HARBOR and CATT analyses, the presence of MA was not associated with worse visual acuity outcomes at 2 years in comparison with eyes without detectable macular atrophy $[61,62]$. 
Fig. 4. Multimodal imaging from a patient with macular atrophy. The right eye of a patient with neovascular AMD complicated with large MA. The green arrow in the near-IR reflectance image (a) illustrates the location and direction of the structural OCT-B scan that demonstrates the presence of macular atrophy. b FAF shows a large hypofluorescent area at the posterior pole with fine granular hyperautofluorescence in the perilesional zone. c Multicolor image shows a large area of atrophy which denudes the underlying choroid. d SDOCT shows a large area of RPE loss, absence of ellipsoid zone and external limiting membrane. AMD, age-related macular degeneration; RPE, retinal pigment epithelium; OCT, optical coherence tomography; FAF, fundus autofluorescence; MA, macular atrophy; IR, infrared.

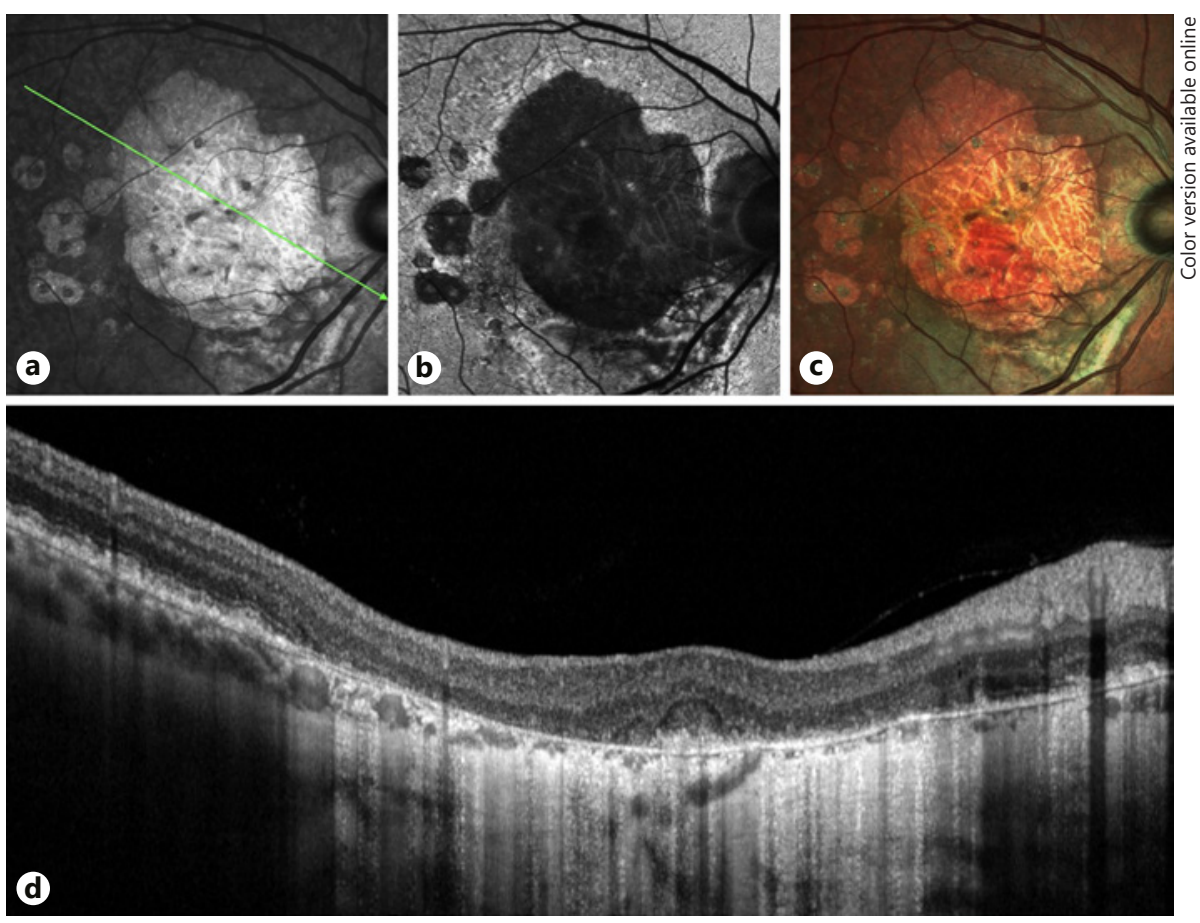

Conversely, MA in the setting of neovascular AMD has been proposed to occur because of interferences with basal VEGF levels owing to anti-VEGF therapy [61]. To date, current evidence suggests that there is not a significant association between anti-VEGF therapy and atrophy development. In the HARBOR study [61], eyes treated with more injections were not associated with a higher rate of MA development considering the PRN treatment arms. Furthermore, although authors noted a trend of increased incidence of MA among patients treated monthly, this difference did not reach a statistical significance.

Another possible explanation is that atrophy is the natural result of MNV regression and fibrocellular replacement with subsequent contraction of the RPE and photoreceptor nourishment [63]. A post hoc analysis of the TREX-AMD trial displayed that MA at 18 months tends to develop in regions co-localizing with MNV at baseline, the latter finding suggesting that the MNV regression and fibrous replacement rather than the anti-VEGF therapy may be an important contributor to MA development [64]. Finally, the RIVAL study showed no statistically significant differences between aflibercept and ranibizumab in terms of MA development or growth over a period of 2 years [65].

Current literature identified numerous factors and phenotypes associated with an increased risk of MA, including fellow eye atrophy (hazard ratio [HR] 2.02; 95\% confidence interval [CI] 1.42-2.87), the presence of intraretinal cysts (HR 2.45; 95\% CI 1.76-3.42) and age (HR, $1.09,95 \%$ CI 1.0027-1.19) [61]. In addition, foveal atrophy may be associated with subretinal drusenoid deposits, type 3 phenotype and frequency of injections [59].

Conversely, the presence of subretinal fluid at baseline was associated with a reduced risk of MA at 2 years ( $\mathrm{HR}$, 0.50; 95\% CI 0.33-0.74) [61]. The latter finding of subretinal fluid as a protective factor for MA was supposed to be secondary to a persistent (and leaking) MNV nourishing the outer retina and RPE. The FLUID trial has recently further investigated the hypothesis that the presence of a small quantity of subretinal fluid does not affect visual acuity outcomes [66]. In the latter study, participants were treated with a TREX regimen and were randomized to receive ranibizumab monthly until either complete resolution of subretinal and intraretinal fluid or resolution of intraretinal fluid only (except for subretinal fluid $>200 \mu \mathrm{m}$ at the foveal center) before extending treatment intervals. At 2 years of follow-up, visual acuity was noninferior with a TREX regimen that tolerated some SRF compared with a TREX regimen with complete SRF resolution. Taking all these results together, it may be suggested that persistence of a small amount of subretinal fluid is not associated with an increased risk of MA development and does not affect long-term visual outcomes. 

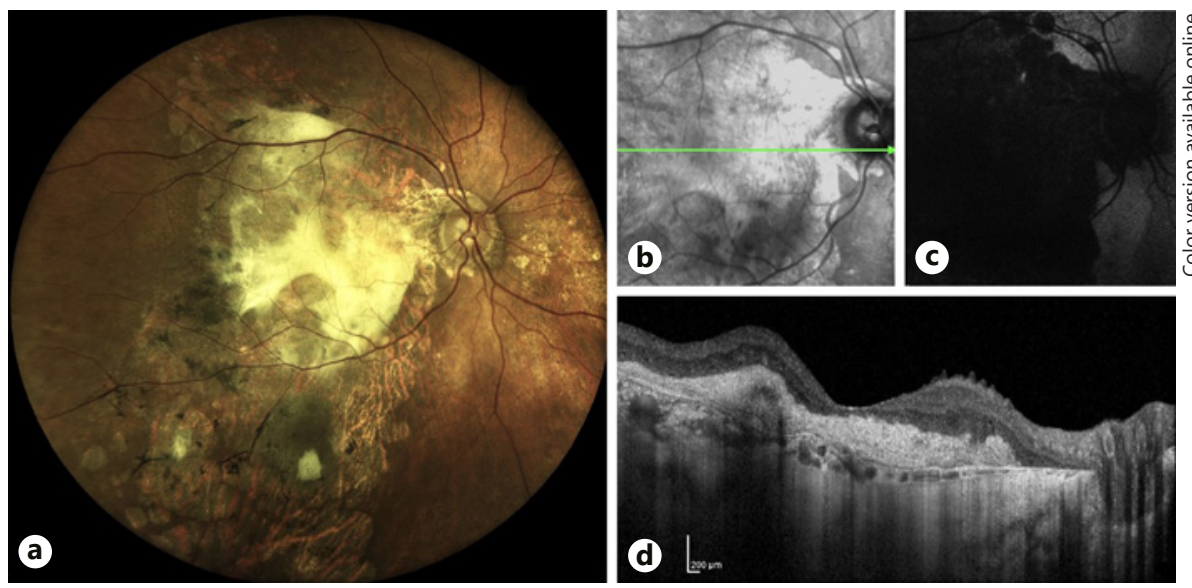

Fig. 5. Multimodal imaging from a patient with macular scar. The right eye of a patient with neovascular AMD complicated with large macular scar. a Color image shows a large yellowish lesion involving the posterior pole and the optic nerve head. The green arrow in the near-IR reflectance image (b) illustrates the location and direction of the structural OCT-B scan. c FAF shows a large hypofluorescent area at the posterior pole. d SD-OCT scan demonstrates the presence of a subretinal scar associated with degeneration of the external limiting membrane and ellipsoid layer. AMD, age-related macular degeneration; OCT, optical coherence tomography; FAF, fundus autofluorescence; IR, infrared.
Currently, there are no approved treatments for MA in neovascular AMD. Although many promising clinical trials have been performed in eyes with MA, most are restricted to forms without MNV. However, new treatments including viral vectors for subretinal gene therapy and RPE patches have been recently investigated for forms of MA developing in the setting of neovascular AMD [67-70].

\section{Macular Scar}

Macular scar formation has been identified as one of the main predictors of visual function in eyes with exudative neovascular AMD as eyes with this complication are frequently characterized by a visual acuity lower than 20/200 Snellen (shown in Fig. 5) [71]. Neovascular (or fibrovascular) lesions are typically composed of a fibrous and vascular component [72]. However, an increase in the fibrous component may result in the development of a macular scar, also accompanied by a regression of the pathological vessels [73].

A macular scar (also identified as fibrocellular lesion) [74] is typically described as a well-demarcated white or yellow lesion on fundus biomicroscopy [75]. Hyperpigmented areas may be present depending on the degree of RPE hyperplasia. This lesion is characteristically hyperreflective on infrared and OCT images [63], with hyperfluorescence due to tissue staining or blocked fluorescence of the underlying choroid in fluorescein angiograms $[76,77]$.

Complications in Exudative Neovascular

Age-Related Macular Degeneration
Hence, during anti-VEGF therapy, exudative neovascular AMD eyes may reach remission (no subretinal hemorrhage, sign of fluid in or under the retina, and no treatment for at least 6 months) because of absence of neovascular leakage (fibrovascular phenotype) or alternatively for the development of a macular scar (fibrocellular phenotype). These 2 phenotypes were morphologically and functionally compared in eyes with MNV treated with anti-VEGF and with persistent absence of neovascular activity [63]. In the latter study, the authors demonstrated a significant association between RPE atrophy and the presence of a fibrocellular scar, even after accounting for confounding factors, such as type of MNV and number of previous anti-VEGF injections. Using OCT angiography, the latter study also demonstrated that the fibrocellular phenotype is characterized by a reduced perfusion of the pathological vessels in comparison with the fibrovascular phenotype. These results strongly support the evidence that an increase in the fibrous component is accompanied by a decrease in vascular cellularity, suggesting that macular scar may evolve along with regression of the pathologic vessels $[78,79]$. Finally, this study further confirmed that eyes with macular scar (fibrocellular phenotype) had a significant worse visual acuity and macular sensitivity, the latter quantified using microperimetry. Given that choroidal neovessels may represent compensatory nourishment to hypoxic outer retinal cells, this evidence advice that a neovascular regression and fibrocellular replacement may reduce this nourishment and con-

Ophthalmologica 2021;244:512-522 
sequently cause RPE atrophy. These findings suggest that maturation of vessels rather than their abolishment should be considered as the finest goal in the treatment of exudative neovascular AMD.

Although anti-VEGF agents are still a first-line treatment option for patients with wet AMD, novel therapeutic strategies have been investigated to reduce the treatment burden due to frequent dosing and to reduce atrophy and fibrocellular replacement (macular scar). To date, Faricimab is the most advanced agent targeting the Ang-Tie pathway, whose upregulation is thought to drive vessel destabilization and inflammatory signaling. Preclinical studies have shown that the dual inhibition of VEGF A and Ang-2 may be more effective in reducing pathological vascular permeability and preserving neovessels, rather than resulting in a neovascular shrinking that may finally result in the development of a macular scar [80-82].

\section{Conclusions}

Neovascular exudative AMD is a common disorder that may lead to a significant impairment in visual function. While anti-VEGF therapy has significantly improved visual outcomes of patients with this disease, complications may occur including subretinal massive hemorrhage, vitreous hemorrhage, RPE tear, MA, and macular scar. These complications may significantly impact on short- and long-term visual outcomes. Therefore, their recognition is relevant for a proper management.
More importantly, although most of these complications are still not properly treatable, new therapeutic options may be available in the near future.

\section{Conflict of Interest Statement}

Francesco Bandello is a consultant for Alcon (Fort Worth, TX, USA), Alimera Sciences (Alpharetta, GA, USA), Allergan Inc (Irvine, CA, USA), Farmila-Thea (Clermont-Ferrand, France), Bayer Shering-Pharma (Berlin, Germany), Bausch And Lomb (Rochester, NY, USA), Genentech (San Francisco, CA, USA), Hoffmann-La-Roche (Basel, Switzerland), Novagali Pharma (Évry, France), Novartis (Basel, Switzerland), Sanofi-Aventis (Paris, France), Thrombogenics (Heverlee, Belgium), Zeiss (Dublin, OH, USA). Giuseppe Querques is a consultant for Alimera Sciences (Alpharetta, GA, USA), Allergan Inc (Irvine, CA, USA), Amgen (Thousand Oaks,USA), Bayer Shering-Pharma (Berlin, Germany), Heidelberg (Germany), KBH (Chengdu; China), LEH Pharma (London, UK), Lumithera (Poulsbo, WA, USA), Novartis (Basel, Switzerland), Sandoz (Berlin, Germany), Sifi (Catania, Italy), Sooft-Fidea (Abano, Italy), Zeiss (Dublin, $\mathrm{OH}, \mathrm{USA}$ ). The other authors have no disclosures.

\section{Funding Sources}

The authors did not receive any funding for this article.

\section{Author Contributions}

All named authors meet the International Committee of Medical Journal Editors (ICMJE) criteria for authorship for this article, take responsibility for the integrity of the work as a whole, and gave their approval for this version to be published.

\section{References}

1 Lim LL, Guymer RH. Age-related macular degeneration. Garner Klintworth's Pathobiol Ocul Dis. 2007:371-92.

2 Wong WL, Su X, Li X, Cheung CM, Klein R, Cheng CY, et al. Global prevalence of age-related macular degeneration and disease burden projection for 2020 and 2040: a systematic review and meta-analysis. Lancet Glob Health. 2014;2(2):e106-16.

3 Spaide RF, Jaffe GJ, Sarraf D, Freund KB, Sadda SR, Staurenghi G, et al. Consensus nomenclature for reporting neovascular age-related macular degeneration data: consensus on neovascular age-related macular degeneration nomenclature study group. Ophthalmology. 2020;127(5):616-36.

4 Ferris FL, Wilkinson CP, Bird A, Chakravarthy U, Chew E, Csaky K, et al. Clinical classification of age-related macular degeneration. Ophthalmology. 2013 Apr;120(4):844-51.
5 Solomon SD, Lindsley K, Vedula SS, Krzystolik MG, Hawkins BS. Anti-vascular endothelial growth factor for neovascular age-related macular degeneration. Cochrane Database Syst Rev. 2019;3(3):CD005139.

6 Tufail A, Patel PJ, Egan C, Hykin P, Da Cruz L, Gregor Z, et al. Bevacizumab for neovascular age related macular degeneration (ABC trial): multicentre randomised double masked study. BMJ. 2010;340(7761):c2459.

7 Sacu S, Michels S, Prager F, Weigert G, Dunavoelgyi $\mathrm{R}$, Geitzenauer W, et al. Randomised clinical trial of intravitreal avastin versus photodynamic therapy and intravitreal triamcinolone: long-term results. Eye. 2009;23(12): 2223-7.
8 Regillo CD, Brown DM, Abraham P, Yue H, Ianchulev T, Schneider S, et al. Randomized, double-masked, sham-controlled trial of ranibizumab for neovascular age-related macular degeneration: PIER study year 1 . Am J Ophthalmol. 2008;145(2):239.

9 Maberley D. Pegaptanib for neovascular agerelated macular degeneration. Issues Emerg Health Technol. 2005;76:1-4.

10 Brown DM, Kaiser PK, Michels M, Soubrane G, Heier JS, Kim RY, et al. Ranibizumab versus verteporfin for neovascular age-related macular degeneration. N Engl J Med. 2006; 355(14):1432-44.

11 Seddon JM, McLeod DS, Bhutto IA, Villalonga MB, Silver RE, Wenick AS, et al. Histopathological insights into choroidal vascular loss in clinically documented cases of agerelated macular degeneration. JAMA Ophthalmol. 2016 Sep. 
12 Curcio CA, Presley JB, Malek G, Medeiros NE, Avery DV, Kruth HS. Esterified and unesterified cholesterol in drusen and basal deposits of eyes with age-related maculopathy. Exp Eye Res. 2005 Dec;81(6):731-41.

13 McLeod DS, Grebe R, Bhutto I, Merges C, Baba T, Lutty GA. Relationship between RPE and choriocapillaris in age-related macular degeneration. Invest Ophthalmol Vis Sci. 2009 Oct;50(10):4982-91.

14 Bressler NM, Bressler SB, Fine SL. Age-related macular degeneration. Surv Ophthalmol. 1988;32(6):375-413.

15 Fine AM, Elman MJ, Ebert JE, Prestia PA, Starr JS, Fine SL. Earliest symptoms caused by neovascular membranes in the macula. Arch Ophthalmol. 1986;104(4):513.

16 Bressler NM, Chang TS, Suñer IJ, Fine JT, Dolan CM, Ward J, et al. Vision-related function after ranibizumab treatment by better- or worse-seeing eye. clinical trial results from marina and anchor. Ophthalmology. 2010; 117(4):747.

17 Yannuzzi LA. A modified Amsler grid. A selfassessment test for patients with macular disease. Ophthalmology. 1982 Feb;89(2):157-9.

18 Martin DF, Maguire MG, Fine SL, Ying GS, Jaffe GJ, Grunwald JE, et al. Ranibizumab and bevacizumab for treatment of neovascular age-related macular degeneration: two-year results. Ophthalmology. 2012;119(7):1388.

19 Busbee BG, Ho AC, Brown DM, Heier JS, Suñer IJ, Li Z, et al. Twelve-month efficacy and safety of $0.5 \mathrm{mg}$ or $2.0 \mathrm{mg}$ ranibizumab in patients with subfoveal neovascular age-related macular degeneration. Ophthalmology. 2013; 120(5):1046-56.

20 Chakravarthy U, Harding SP, Rogers CA, Downes SM, Lotery AJ, Culliford LA, et al. Alternative treatments to inhibit VEGF in age-related choroidal neovascularisation: 2 -year findings of the IVAN randomised controlled trial. Lancet. 2013;382(9900):1258.

21 Gupta OP, Shienbaum G, Patel AH, Fecarotta C, Kaiser RS, Regillo CD. A treat and extend regimen using ranibizumab for neovascular age-related macular degeneration: clinical and economic impact. Ophthalmology. 2010; 117(11):2134

22 Shienbaum G, Gupta OP, Fecarotta C, Patel AH, Kaiser RS, Regillo CD. Bevacizumab for neovascular age-related macular degeneration using a treat-and-extend regimen: clinical and economic impact. Am J Ophthalmol. 2012;153(3):468.

23 Silva R, Berta A, Larsen M, Macfadden W, Feller C, Monés J. Treat-and-extend versus monthly regimen in neovascular age-related macular degeneration: results with ranibizumab from the trend study. Ophthalmology. 2018;125(1):57.

24 Berg K, Hadzalic E, Gjertsen I, Forsaa V, Berger LH, Kinge B, et al. Ranibizumab or bevacizumab for neovascular age-related macular degeneration according to the lucentis compared to avastin study treat-and-extend protocol: two-year results. Ophthalmology. 2016;123(1):51.
25 Wykoff CC, Croft DE, Brown DM, Wang R, Payne JF, Clark L, et al. Prospective trial of treat-and-extend versus monthly dosing for neovascular age-related macular degeneration: TREX-AMD 1-year results. Ophthalmology. 2015;122(12):2514-22..

26 Wykoff CC, Ou WC, Brown DM, Croft DE, Wang R, Payne JF, et al. Randomized trial of treat-and-extend versus monthly dosing for neovascular age-related macular degeneration: 2-year results of the TREX-AMD study. Ophthalmol Retina. 2017 Jul;1(4):314-21.

27 Stanescu-Segall D, Balta F, Jackson TL. Submacular hemorrhage in neovascular age-related macular degeneration: a synthesis of the literature. Surv Ophthalmol. 2016;61(1):18-32.

28 El Baba F, Jarrett WH, Harbin TS, Fine SL, Michels RG, Schachat AP, et al. Massive hemorrhage complicating age-related macular degeneration: clinicopathologic correlation and role of anticoagulants. Ophthalmology. 1986; 93(12):1581-92.

29 Ying GS, Maguire MG, Daniel E, Grunwald JE, Ahmed O, Martin DF. Association between antiplatelet or anticoagulant drugs and retinal or subretinal hemorrhage in the comparison of age-related macular degeneration treatments trials. Ophthalmology. 2016; 123(2):352.

30 Argon laser photocoagulation for neovascular maculopathy: five-year results from randomized clinical trials. Arch Ophthalmol. 1991;109(8):1109.

31 Laser photocoagulation of subfoveal neovascular lesions of age-related macular degeneration: updated findings from two clinical trials. Arch Ophthalmol. 1993;111(9):1200.

32 Hawkins BS. Laser photocoagulation of subfoveal recurrent neovascular lesions in agerelated macular degeneration: results of a randomized clinical trial. Arch Ophthalmol. 1991;109(9):1232.

33 Avery RL, Fekrat S, Hawkins BS, Bressler NM. Natural history of subfoveal subretinal hemorrhage in age-related macular degeneration. Retina. 1996;16(3):183-9.

34 Toth CA, Morse LS, Hjelmeland LM, Landers MB. Fibrin directs early retinal damage after experimental subretinal hemorrhage. Arch Ophthalmol. 1991;109(5):723-9.

35 Bennett SR, Folk JC, Blodi CF, Klugman M. Factors prognostic of visual outcome in patients with subretinal hemorrhage. Am J Ophthalmol. 1990 Jan;109(1):33-7.

36 Yiu G, Mahmoud TH. Subretinal hemorrhage. Dev Ophthalmol. 2014;54:213-22.

37 Sacu S, Stifter E, Vécsei-Marlovits PV, Michels S, Schütze C, Prünte C, et al. Management of extensive subfoveal haemorrhage secondary to neovascular age-related macular degeneration. Eye. 2009;23(6):1404-10.

38 Hassan AS, Johnson MW, Schneiderman TE, Regillo CD, Tornambe PE, Poliner LS, et al. Management of submacular hemorrhage with intravitreous tissue plasminogen activator injection and pneumatic displacement. Ophthalmology. 1999;106(10):1900-7.
39 Chen CY, Hooper C, Chiu D, Chamberlain M, Karia N, Heriot WJ. Management of submacular hemorrhage with intravitreal injection of tissue plasminogen activator and expansile gas. Retina. 2007;27(3):321-8.

40 Treumer F, Roider J, Hillenkamp J. Longterm outcome of subretinal coapplication of rtPA and bevacizumab followed by repeated intravitreal anti-VEGF injections for neovascular AMD with submacular haemorrhage. Br J Ophthalmol. 2012;96(5):708-13.

41 Treumer F, Klatt C, Roider J, Hillenkamp J. Subretinal coapplication of recombinant tissue plasminogen activator and bevacizumab for neovascular age-related macular degeneration with submacular haemorrhage. $\mathrm{Br} \mathrm{J}$ Ophthalmol. 2010;94(1):48-53.

42 Kim TY, Kang HG, Choi EY, Koh HJ, Kim SS, Lee JH, et al. Prognostic factors and long-term surgical outcomes for exudative age-related macular degeneration with breakthrough vitreous hemorrhage. Korean J Ophthalmol. 2020;34(4):281-9.

43 Azzolini C, Menchini U, Pece A, Camesasca F, Giuliani V. Age-related macular degeneration and vitreous hemorrhage. Eur J Ophthalmol. 1991;1(3):142-7.

44 Hasegawa T, Otani A, Sasahara M, Gotoh N, Ooto S, Tamura H, et al. Prognostic factors of vitreous hemorrhage secondary to exudative age-related macular degeneration. Am J Ophthalmol. $2010 \mathrm{Feb}$ 1;149(2):322-e1.

45 Kim JH, Kim JW, Kim CG, Lee DW. Longterm clinical course after vitrectomy for breakthrough vitreous hemorrhage secondary to neovascular age-related macular degeneration and polypoidal choroidal vasculopathy. Sci Rep. 2020;10(1):359-9.

46 Jung JH, Lee JK, Lee JE, Oum BS. Results of vitrectomy for breakthrough vitreous hemorrhage associated with age-related macular degeneration and polypoidal choroidal vasculopathy. Retina. 2010;30(6):865-73.

47 Roberts SP, Roberts DK, Stelmack TR. Tears of the retinal pigment epithelium. Clin Eye Vis Care. 1992;4(1):33-8.

48 Chang LK, Sarraf D. Tears of the retinal pigment epithelium: an old problem in a new era. Retina. 2007;27(5):523.

49 Ersoz MG, Karacorlu M, Arf S, Sayman Muslubas I, Hocaoglu M. Retinal pigment epithelium tears: classification, pathogenesis, predictors, and management. Surv Ophthalmol. 2017;62(4):493

50 Mouallem A, Sarraf D, Chen X, Capuano V, Souied EH, Querques G. Double retinal pigment epithelium tears in neovascular age-related macular degeneration. Retina. 2016 Nov;36(11):2197-204.

51 Sastre-Ibáñez M, Martínez-Rubio C, MolinaPallete R, Martínez- López-Corell P, Wu L, Arévalo JF, et al. Retinal pigment epithelial tears. Journal Français d'Ophtalmologie. 2019;42(1):63-72. 
52 Clemens CR, Eter N. Retinal pigment epithelium tears: risk factors, mechanism and therapeutic monitoring. Ophthalmologica. 2016; 235(1):1-9.

53 Doguizi S, Ozdek S. Pigment epithelial tears associated with anti-VEGF therapy: incidence, long-term visual outcome, and relationship with pigment epithelial detachment in age-related macular degeneration. Retina. 2014;34(6):1156-62.

54 Sarraf D, Chan C, Rahimy E, Abraham P. Prospective evaluation of the incidence and risk factors for the development of RPE tears after high-and low-dose ranibizumab therapy. Retina. 2013;33(8):1551.

55 Mukai R, Sato T, Kishi S. Erratum: outsourced cataract surgery and postoperative endophthalmitis. Acta Ophthalmol. 2014;92(5): 2013-5.

56 Querques G, Capuano V, Costanzo E, Corvi F, Querques L, Introini U, et al. Retinal pigment epithelium aperture: a previously unreported finding in the evolution of avascular pigment epithelium detachment. Retina. 2016;36 Suppl 1:S65-S72.

57 Sarraf D, Reddy S, Chiang A, Yu F, Jain A. A new grading system for retinal pigment epithelial tears. Retina. 2010;30(7):1039-45.

58 Moreira CA, Arana LA, Zago RJ. Long-term results of repeated anti-vascular endothelial growth factor therapy in eyes with retinal pigment epithelial tears. Retina. 2013;33(2):27781.

59 Sadda SVR, Guymer R, Monés JM, Tufail A, Jaffe GJ. Anti-vascular endothelial growth factor use and atrophy in neovascular age-related macular degeneration: systematic literature review and expert opinion. Ophthalmology. 2020;127(5):648-59.

60 Abdelfattah NS, Al-Sheikh M, Pitetta S, Mousa A, Sadda SR, Wykoff CC, et al. Macular atrophy in neovascular age-related macular degeneration with monthly versus treat-and-extend ranibizumab: findings from the TREX-AMD trial. Ophthalmology. $2017 \mathrm{Feb}$; 124(2):215-23

61 Sadda SR, Tuomi LL, Ding B, Fung AE, Hopkins JJ. Macular atrophy in the harbor study for neovascular age-related macular degeneration. Ophthalmology. 2018 Jun;125(6): $878-86$.
62 Grunwald JE, Daniel E, Huang J, Ying GS, Maguire MG, Toth CA, et al. Risk of geographic atrophy in the comparison of age-related macular degeneration treatments trials. Ophthalmology. 2014;121(1):150.

63 Querques L, Parravano M, Borrelli E, Chiaravalloti $A$, Tedeschi $M$, Sacconi $R$, et al. Anatomical and functional changes in neovascular AMD in remission: comparison of fibrocellular and fibrovascular phenotypes. $\mathrm{Br}$ J Ophthalmol. 2020;104(1):47-52.

64 Abdelfattah NS, Hariri AH, Al-Sheikh M, Pitetta S, Ebraheem A, Wykoff CC, et al. Topographic correspondence of macular atrophy with choroidal neovascularization in ranibizumab-treated eyes of the TREX-AMD trial. Am J Ophthalmol. 2018 Aug;192:84-90.

65 Gillies MC, Hunyor AP, Arnold JJ, Guymer RH, Wolf S, Pecheur FL, et al. Macular atrophy in neovascular age-related macular degeneration: a randomized clinical trial comparing ranibizumab and aflibercept (RIVAL study). Ophthalmology. 2020 Feb;127(2): 198-210.

66 Guymer RH, Markey CM, McAllister IL, Gillies MC, Hunyor AP, Arnold JJ, et al. Tolerating subretinal fluid in neovascular age-related macular degeneration treated with ranibizumab using a treat-and-extend regimen: fluid study 24-month results. Ophthalmology. 2019;126(5):723.

67 Akyol E, Lotery A. Gene, cell and antibodybased therapies for the treatment of age-related macular degeneration. Biologics. 2020;14: 83-94.

68 Mertz L. Gift of sight: stem-cell patches for wet and dry macular degeneration. IEEE Pulse. 2019;10(2):8-13.

69 Nebbioso M, Lambiase A, Cerini A, Limoli PG, La Cava M, Greco A. Therapeutic approaches with intravitreal injections in geographic atrophy secondary to age-related macular degeneration: current drugs and potential molecules. Int J Mol Sci. 2019;20(7): 1693.

70 Kashani AH, Uang J, Mert M, Rahhal F, Chan C, Avery RL, et al. Surgical method for implantation of a biosynthetic retinal pigment epithelium monolayer for geographic atrophy: experience from a phase $1 / 2 \mathrm{a}$ study. Ophthalmol Retina. 2020;4(3):264-73.
71 Hogg R, Curry E, Muldrew A, Winder J, Stevenson $\mathrm{M}, \mathrm{McCl}$ ure $\mathrm{M}$, et al. Identification of lesion components that influence visual function in age related macular degeneration. $\mathrm{Br} \mathrm{J}$ Ophthalmol. 2003;87(5):609-14.

72 Friedlander M. Fibrosis and diseases of the eye. J Clin Invest. 2007;117:576-86.

73 Schmidt-Erfurth U, Waldstein SM. A paradigm shift in imaging biomarkers in neovascular age-related macular degeneration. Prog Retin Eye Res. 2016;50:1-24.

74 Grossniklaus HE, Green WR. Histopathologic and ultrastructural findings of surgically excised choroidal neovascularization. Submacular surgery trials research group. Arch Ophthalmol. 1998;116(6):745-9.

75 Toth CA, Tai V, Pistilli M, Chiu SJ, Winter K, Daniel E, et al. HHS Public Access; 2020.

76 Casalino G, Stevenson MR, Bandello F, Chakravarthy U. Tomographic biomarkers predicting progression to fibrosis in treated neovascular age-related macular degeneration: a multimodal imaging study. Ophthalmol Retina. 2018;2(5):451-61.

77 Daniel E, Toth CA, Grunwald JE, Glenn J, Martin DF, Fine SL, et al. NIH public access; 2015.

78 Hinton DR, He S, Lopez PF. Apoptosis in surgically excised choroidal neovascular membranes in age-related macular degeneration. Arch Ophthalmol. 1998;116(2):203-9.

79 Ishikawa K, Kannan R, Hinton DR. Molecular mechanisms of subretinal fibrosis in age-related macular degeneration. Exp Eye Res. 2014;142:19-25.

80 Samanta A, Aziz AA, Jhingan M, Singh SR Khanani A, Chhablani J. Emerging therapies in neovascular age-related macular degeneration in 2020. Asia Pac J Ophthalmol. 2020; 9(3):250-9.

81 Baumal CR. Wet age-related macular degeneration: treatment advances to reduce the injection burden. Am J Manag Care. 2020 May; 26(5):S103-11.

82 Nicolò M, Ferro Desideri L, Vagge A, Traverso CE. Faricimab: an investigational agent targeting the Tie-2/angiopoietin pathway and VEGF-A for the treatment of retinal diseases. Expert Opin Investig Drugs. 2021;30(3):193200 